\title{
Coronal X-ray emission and planetary irradiation in HD 209458
}

\author{
S. Czesla, M. Salz, P. C. Schneider, M. Mittag, and J. H. M. M. Schmitt
}

Hamburger Sternwarte, Universität Hamburg, Gojenbergsweg 112, 21029 Hamburg, Germany

e-mail: stefan.czesla@hs.uni-hamburg.de

Received 20 June 2017 / Accepted 10 August 2017

\begin{abstract}
HD 209458 is one of the benchmark objects in the study of hot Jupiter atmospheres and their evaporation through planetary winds. The expansion of the planetary atmosphere is thought to be driven by high-energy extreme ultraviolet (EUV) and X-ray irradiation. We obtained new Chandra High Resolution Camera (HRC-I) data, which unequivocally show that HD 209458 is an X-ray source. Combining these data with archival XMM-Newton observations, we find that the corona of HD 209458 is characterized by a temperature of about $1 \mathrm{MK}$ and an emission measure of $7 \times 10^{49} \mathrm{~cm}^{-3}$, yielding an X-ray luminosity of $1.6 \times 10^{27} \mathrm{erg} \mathrm{s}^{-1}$ in the $0.124-2.48 \mathrm{keV}$ band. HD 209458 is an inactive star that has a coronal temperature comparable to that of the inactive Sun but that has a larger emission measure. At this level of activity, the planetary high-energy emission is sufficient to support mass loss at a rate of a few times $10^{10} \mathrm{~g} \mathrm{~s}^{-1}$.
\end{abstract}

Key words. stars: individual: HD 209458 - stars: coronae - stars: activity - X-rays: stars - planets and satellites: gaseous planets

\section{Introduction}

Planets orbiting their host stars at close proximity are thought to undergo mass loss through planetary winds driven by stellar X-ray and extreme ultraviolet (EUV) irradiation (e.g., Salz et al. 2016a,b). This may even affect planetary evolution (Sanz-Forcada et al. 2010) and thus, precise knowledge of the stellar high-energy spectrum is critical to understand both the stars and their planets.

One of the key systems in which planetary mass loss can be studied to date is HD 209458. In fact, the G0 dwarf HD 209458 is one of the optically brightest stars known to host a transiting hot Jupiter, which orbits the star every $3.5 \mathrm{~d}$ at a distance of about 0.047 AU (Charbonneau et al. 2000; Henry et al. 2000). HD 209458 b was among the first planets for which mass loss was detected by means of enhanced in-transit absorption in the Ly $\alpha$ line (Vidal-Madjar et al. 2003). Subsequently, further elements such as oxygen and carbon were reported in the planetary wind (Vidal-Madjar et al. 2004).

The distance to the HD 209458 system was determined by Gaia to be $48.9 \pm 0.5$ pc (DR1; Gaia Collaboration 2016a,b). Using hydrogen Lyman $\alpha$ line observations, Wood et al. (2005) determined an interstellar H I column density of $2.3 \times 10^{18} \mathrm{~cm}^{-2}$ toward HD 209458. The star is slightly larger than the Sun (1.2 $R_{\odot}$; Boyajian et al. 2015; del Burgo \& Allende Prieto 2016), shows solar iron abundance $(\mathrm{Fe} / \mathrm{H}=0.02 \pm 0.03$, Santos et al. 2004), and is a bona fide single (Raghavan et al. 2006; Daemgen et al. 2009).

According to its projected rotation velocity, the rotation period of HD 209458 is $14.4 \pm 2.1 \mathrm{~d}$, assuming the system is aligned, which is consistent with measurements of the RossiterMcLaughlin effect (Winn et al. 2005). Silva-Valio (2008) favor a somewhat shorter rotation period of $11.4 \mathrm{~d}$ based on in-transit starspot observations. According to Bonfanti et al. (2016), the age of HD 209458 is about 4.4 Gyr, i.e., essentially the age of the Sun. Yet, age estimates for HD 209458 differ widely from around $2 \mathrm{Gyr}$, as determined by Maxted et al. (2015) using gyrochronology and isochrones, to $6.5 \mathrm{Gyr}$, as reported by Boyajian et al. (2015) based on evolutionary modeling, however, with a large uncertainty of nearly 3 Gyr.

Although HD 209458 rotates at about twice the solar rate, it is a rather inactive star. Henry et al. (2000) report a $\log R_{H K}^{\prime}$ value of -4.93. Between 2004 and the end of 2009, HD 209458 was observed in the context of the California Planet Search (Isaacson \& Fischer 2010). During this period, the star showed a continuously low activity level with $\log R_{H K}^{\prime}$ values ranging between -4.930 and -4.974 , i.e., it remained close to the minimum solar activity level of $\log R_{H K}^{\prime} \approx-4.96$ (Mamajek \& Hillenbrand 2008). This is also consistent with the value of -4.97 reported by Knutson et al. (2010). From the coadded spectrum obtained between Aug. 2013 and Aug. 2014 by the TIGRE telescope (Mittag et al. 2011; Schmitt et al. 2014), we measured a Mount Wilson S-index of $0.167 \pm 0.006$ (e.g., Mittag et al. 2016). Applying the calibration by Noyes et al. (1984), this value can be converted into $\log \left(R_{H K}^{\prime}\right)=-4.91 \pm 0.03$, which is comparable to the previously determined values.

Despite its critical role in planetary mass loss, the X-ray spectrum and luminosity of HD 209458 remain controversial. Sanz-Forcada et al. (2011) determined an upper limit of $2.5 \times 10^{26} \mathrm{erg} \mathrm{s}^{-1}$ for the X-ray luminosity of HD 209458 (0.12-2.48 keV band) using XMM-Newton data and assuming a coronal temperature of $2 \mathrm{MK}$. The $3 \mathrm{XMM}$ catalog lists fluxes for a source with a position about two arcseconds from that of HD 209458 (Rosen et al. 2016). Adopting the distance of HD 209458, the reported flux yields an X-ray source with a luminosity of $(6.7 \pm 3.8) \times 10^{26} \mathrm{erg} \mathrm{s}^{-1}$ in the $0.2-12 \mathrm{keV}$ band (Louden et al. 2017). The reconstruction of the differential emission measure presented by Louden et al. (2017) yields an X-ray luminosity of $(1.2 \pm 0.2) \times 10^{27} \mathrm{erg} \mathrm{s}^{-1}$ in the $0.124-2.48 \mathrm{keV}$ band. The authors used the $3 \mathrm{XMM}$ catalog fluxes to constrain their model. According to their analysis, the mass-loss rate of the planet HD $209458 \mathrm{~b}$ is $(3.8 \pm 0.2) \times 10^{10} \mathrm{~g} \mathrm{~s}^{-1}$.

Notably, all of the above estimates of the soft X-ray flux are fully or partly based on the available XMM-Newton data, 


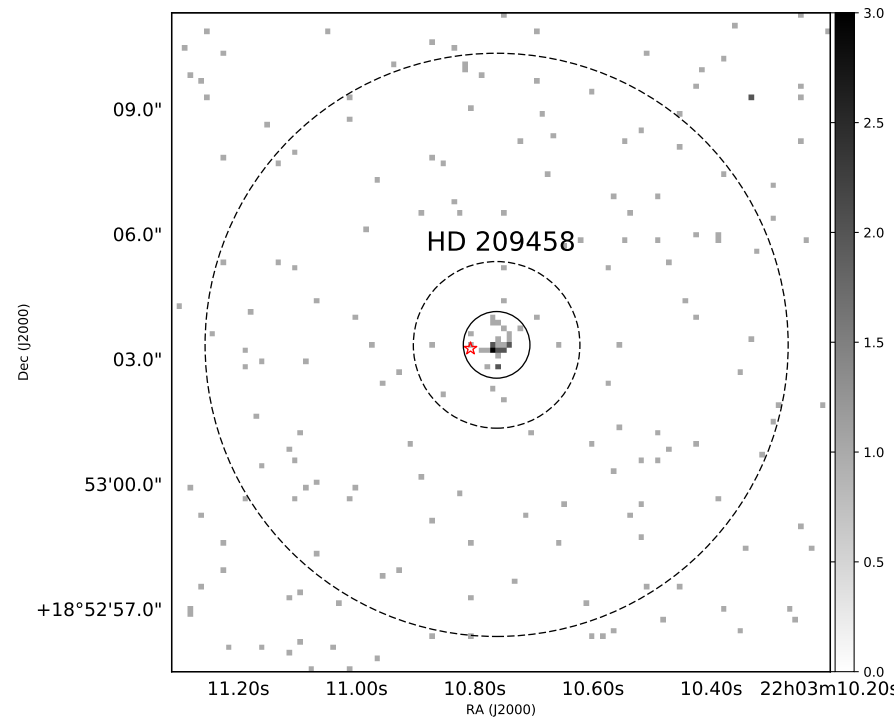

Fig. 1. Chandra HRC-I image of HD 209458 with our circular source region, the background annulus (dashed), and a star symbol indicating the nominal, proper-motion corrected year 2016.5 position from Gaia.

which suffer from considerable background contamination; this complicates the analysis of a weak and soft source such as HD 209458. Therefore, we obtained a new Chandra High Resolution Camera (HRC-I) observation to constrain the soft X-ray flux of HD 209458 more stringently.

\section{Data analysis}

We carry out a detailed analysis of our new Chandra HRC-I data, the available archival XMM-Newton observations and, finally, present a combined analysis of both data sets.

\subsection{Chandra HRC-I data}

Chandra observed HD 209458 on June 17, 2016, for 28.9 ks using the HRC-I (observation ID 16667). Our analysis is based on the pipeline-produced event files and CIAO in version 4.8. While the HRC-I does not provide any notable energy resolution ${ }^{1}$, its sensitivity extends to photon energies of $80 \mathrm{eV}$, which allows us to study soft X-ray sources. In Fig. 1, we show the HRC-I image of the surroundings of HD 209458.

We identified a clear X-ray source close to the nominal position of HD 209458 (see Fig. 1). As our source region, we used a circle with a radius of $0.8^{\prime \prime}$ also indicated in Fig. 1. Using the CIAO task psffrac, we estimated that this region contains $93 \%$ of the photons with an energy of $200 \mathrm{eV}$. We first centered the source region on the visual position of the source and then refined the position by determining the centroid of the photon distribution (see Table 1). In the final source region, a total of 29 counts were recorded.

The centroid position of the X-ray source is offset by $(0.6 \pm$ $0.2)^{\prime \prime}$ toward the west with respect to the proper-motion corrected optical position of HD 209458 determined by Gaia (DR1; Gaia Collaboration 2016a,b). This offset is within the accuracy range of the Chandra aspect solution ${ }^{2}$, therefore we are

1 See, e.g., the report "Spectral Response of the HRC-I" by V. Kashyap and J. Posson-Brown at http: //cxc. cfa.harvard. edu/ ccr/proceedings/05_proc/presentations/kashyap2/

2 http://cxc.harvard.edu/proposer/POG/html/chap5.html
Table 1. Source coordinates determined from HRC-I data and obtained from Gaia and the USNO-B1 catalog.

\begin{tabular}{lcc}
\hline \hline Source & RA [deg] & Dec [deg] \\
\hline \multicolumn{3}{c}{ HD 209458 } \\
HRC-I & 330.794845 & 18.884262 \\
Gaia (2015) & 330.795018 & 18.884244 \\
\hline \multicolumn{3}{c}{ Reference source } \\
HRC-I & 330.801708 & 18.943287 \\
USNO-B1 & 330.801887 & 18.943328 \\
\hline
\end{tabular}

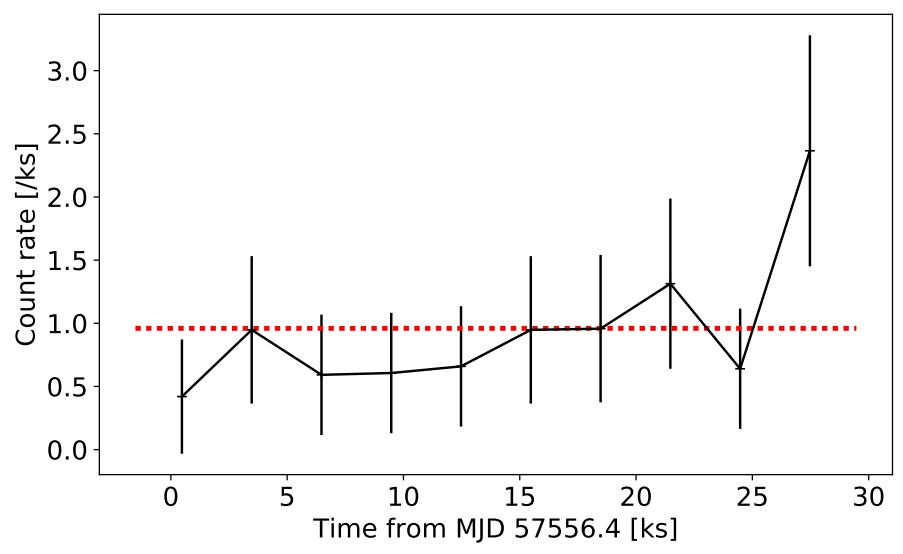

Fig. 2. Background-subtracted HRC-I light curve of HD 209458 with a temporal binning of $3 \mathrm{ks}$ along with the mean count rate (red, dotted).

confident that the X-ray source is associated with the bright star HD 209458 (see also Sect. 2.1).

We estimate 1.4 background counts in the source region by considering an annulus with the same centroid and limiting radii of $2^{\prime \prime}$ and $7 "$. Given the exposure time of $28.9 \mathrm{ks}$, this yields a net source count rate of $(0.96 \pm 0.19) \mathrm{cts} \mathrm{ks}^{-1}$. The HRC-I light curve of HD 209458 shows no obvious variability such as a flare and is compatible with a constant flux (see Fig. 2).

Although we are confident that the small positional offset between the X-ray source and the optical position of HD 209458 is instrumental, this can be validated by finding other X-ray sources in the field with known optical counterparts. Therefore, we searched for reference X-ray sources using the celldetect algorithm. The closest appropriate X-ray source is located 3.6' nearly due north of HD 209458. The centroid position of the source with about 40 registered net counts corresponds to a faint red object in the USNO-B1 catalog (source ID 089-0583168, $R_{2}=19.9$ mag; Monet et al. 2003). This source is located $0.6^{\prime \prime}$ nearly due east of the X-ray position, which supports the contention that the offset seen for HD 209458 is in fact due to a slight error in the aspect solution.

\subsection{XMM-Newton data}

XMM-Newton observed HD 209458 in the years 2000 and 2006

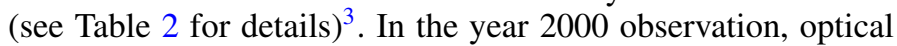
loading caused by the use of the thin filter, leaves only the MOS2 data usable for our analysis.

3 An additional observation in 2003 (Obs. ID 0148200101) is not scientifically usable because of a filter-wheel failure. 
Table 2. XMM-Newton observations of HD 209458.

\begin{tabular}{|c|c|c|c|c|}
\hline $\begin{array}{l}\text { Obs. ID/ } \\
\text { date }\end{array}$ & Camera & Filter & $\begin{array}{l}\text { Duration } \\
{[\mathrm{ks}]}\end{array}$ & $\begin{array}{l}\text { Livetime } \\
{[\mathrm{ks}]}\end{array}$ \\
\hline 0130920101 & MOS1 & $\operatorname{thin}^{a}$ & 17.3 & \\
\hline 2000-Nov.-29 & $\begin{array}{l}\text { MOS2 } \\
\text { pn }\end{array}$ & $\begin{array}{l}\text { thick } \\
\text { thin }^{a}\end{array}$ & $\begin{array}{l}18.1 \\
16.4\end{array}$ & 16.2 \\
\hline 0404450101 & MOS1 & medium & 33.3 & 32.8 \\
\hline 2006-Nov.-14 & $\begin{array}{l}\text { MOS2 } \\
\text { pn }\end{array}$ & $\begin{array}{l}\text { medium } \\
\text { medium }\end{array}$ & $\begin{array}{l}33.3 \\
31.6\end{array}$ & $\begin{array}{l}32.8 \\
26.4\end{array}$ \\
\hline
\end{tabular}

Notes. ${ }^{(a)}$ Corrupted by optical loading.

We reduced the data with the Scientific Analysis System (SAS) in version 16.0.0 . In the reduction, we used standard reduction recipes. We used the epreject task to improve the pn energy scale particularly at low photons energies. The highenergy background of the observations is well behaved with only the 2006 pn observation showing a weak, approximately $2 \mathrm{ks}$ long background flare, which is excluded from our analysis.

To test whether X-ray emission of HD 209458 can be detected in the XMM-Newton data, we carried out a detection experiment. For all scientifically usable exposures, we defined a circular source region with a radius of $15^{\prime \prime}$ centered on the source position as determined from the optical monitor images; none of these is more than $2.5^{\prime \prime}$ from the nominal optical position. We then extracted the counts in these source regions in a soft $(0.15-0.5 \mathrm{keV})$ and a hard spectral band $(0.5-1.5 \mathrm{keV})$, which together comprise the bulk of X-ray emission from rather inactive stars such as HD 209458, which is detectable with $X M M$-Newton's EPIC instruments. While no emission at higher photon energies is expected, there may be substantial emission at lower energies that are not covered by XMM-Newton.

After visual inspection of the X-ray images, we defined an annulus-shaped background region with an inner radius of $30^{\prime \prime}$ and an outer radius of $65^{\prime \prime}$ (see Fig. 3). This background region does not cover obvious X-ray sources in the vicinity of HD 209458; also the Chandra HRC-I image does not show any sources in this region (see Sect. 2.1). Only for the 2006 pn observation, the background annulus covers the chip edges. This does not appear to introduce any particular artifacts. Generally, the background in the vicinity of HD 209458 seems to be inhomogeneous so that differently placed circular reference regions yield different rates. Ignorant of the actual source-region background, we consider that the annulus yields an appropriate estimate. The detected source and background counts in the soft and hard band are listed in Table 3. Along with these numbers we list the number of net counts, $n_{\text {net }}$, calculated according to

$n_{\text {net }}=s-f_{\mathrm{a}} b$ and $\sigma_{\text {net }}=\sqrt{s+f_{\mathrm{a}}^{2} b}$,

where $s$ and $b$ are the number of photons detected in the source and background region and $f_{\mathrm{a}}$ the ratio of their areas. Of course, negative values are not physically admissible and a more stringent statistical treatment is applied in Sect. 3.

In the soft band, all individual observations yield a surplus of events in the source region compared to the background. While not overly significant individually, all soft-band results may be combined to yield a total of $36.2 \pm 8.4$ counts, which yields a significant detection in the soft band. In the year 2000 MOS2 observation, we even find a slightly larger excess in the hard band

4 "Users Guide to the XMM-Newton Science Analysis System", Issue 12.0, 2016 (ESA: XMM-Newton SOC). than in the soft band, which is not the case for the year 2006 observations, where only the pn yields a marginally positive net count rate in the hard band.

\subsection{EPIC pn spectral analysis for year 2006}

The year 2006 observation by the XMM-Newton pn camera provides sufficient photons for a coarse spectral analysis, which we carried out using XSPEC (version 12.9.1, Arnaud 1996). In Fig. 4, we show the pn spectrum along with the best-fit onetemperature thermal APEC model (Foster et al. 2012). In the modeling, the interstellar hydrogen column density remained fixed at the value of $2.3 \times 10^{18} \mathrm{~cm}^{-2}$ determined by Wood et al. (2005) and the metallicity is taken to be solar.

The model yields a best-fit temperature of $0.08_{-0.04}^{+0.06} \mathrm{keV}$ with the error indicating the $90 \%$ confidence limits. The best-fit emission measure is $0.9 \times 10^{50} \mathrm{~cm}^{-3}$, which remains rather loosely constrained. In fact, the $90 \%$ confidence limit only provides an upper limit of $21 \times 10^{50} \mathrm{~cm}^{-3}$, which is essentially due to a degeneracy between temperature and emission measure in this range.

\section{Combined HRC-I and XMM-Newton analysis}

At our disposal we find a rather heterogeneous data set. A total of three observational epochs in the years 2000, 2006, and 2016 are available, and the data of no two epochs were obtained with the same instrumental setup.

While the Chandra HRC-I observation yields a clear detection of the X-ray emission, this observation provides no spectral resolution in itself. The latter can be provided by XMM-Newton, the observations of which however, suffer from comparably high background contamination, allowing only a detection of somewhat debatable quality. Nonetheless, all observations provide information on the X-ray emission of HD 209458.

Therefore, we carried out a combined analysis of all three observational epochs. Although we are aware that also the X-ray emission of inactive stars such as HD 209458 and the Sun can change considerably on the timescale of years (e.g., Peres et al. 2000; Robrade et al. 2012; Ayres 2014), the hypothesis in the following is that all observations can be explained by the same physical model with identical coronal properties. Specifically, we assumed an absorbed, single-temperature thermal APEC model with the interstellar absorption column fixed to the known value. Thus, we ended up with two free parameters: viz., the plasma temperature, $T$, and the emission measure, $E M$. Besides spectral variability, the cross-calibration of the instruments introduces an additional uncertainty (e.g., Poppenhäger et al. 2009; Robrade et al. 2012).

We proceeded by using PyXSPEC to derive model count rates as a function of plasma temperature and emission measure for all available observations and the soft and hard spectral bands. The corresponding response files and auxiliary response files were obtained using CIAO and SAS routines, respectively.

We used the source and background counts obtained for the Chandra HRC-I (Sect. 2.1) and the rates obtained for $X M M$-Newton in the soft and hard spectral bands (Table 3). Assuming uniform priors on the emission measure $(E M)$ and plasma temperature, $T$, our posterior is written as

$$
p(E M, T \mid I) \sim \prod_{i} \frac{P\left(b_{i} \mid \lambda_{b_{i}} e_{i}\right)}{\lambda_{b_{i}}} P\left(s_{i} \mid\left(f_{a_{i}} \lambda_{b_{i}}+\lambda_{s_{i}}(E M, T)\right) e_{i}\right),
$$

where the index $i$ is meant to run over all observations and the soft and hard spectral bands with the exception of the HRC-I 

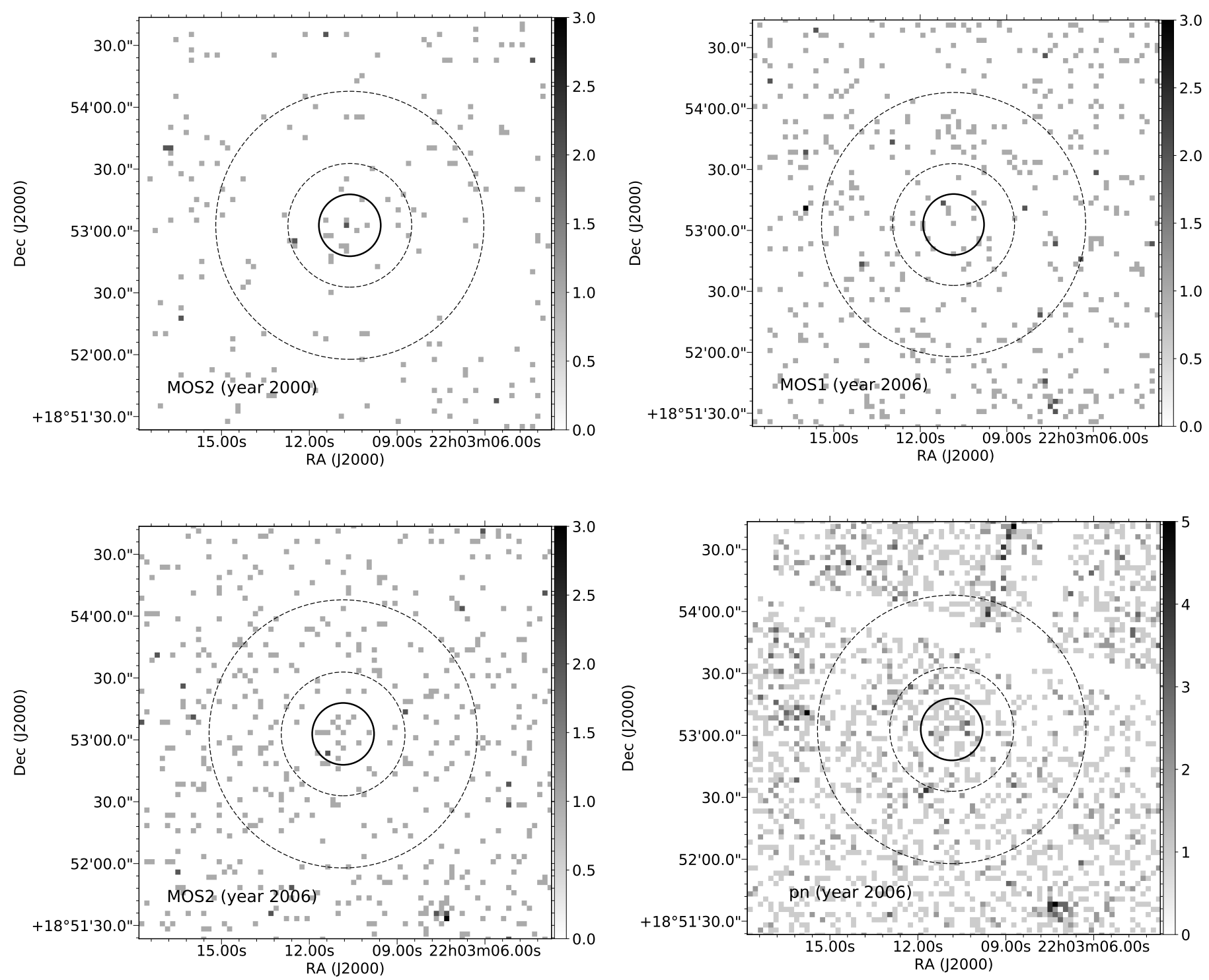

Fig. 3. XMM-Newton X-ray images of HD 209458 and its surroundings in the $0.15-1.5 \mathrm{keV}$ band; EPIC detector and year of observation are indicated in the panels. The solid circle indicates the $15^{\prime \prime}$ source region and the dashed annulus indicates the background region.

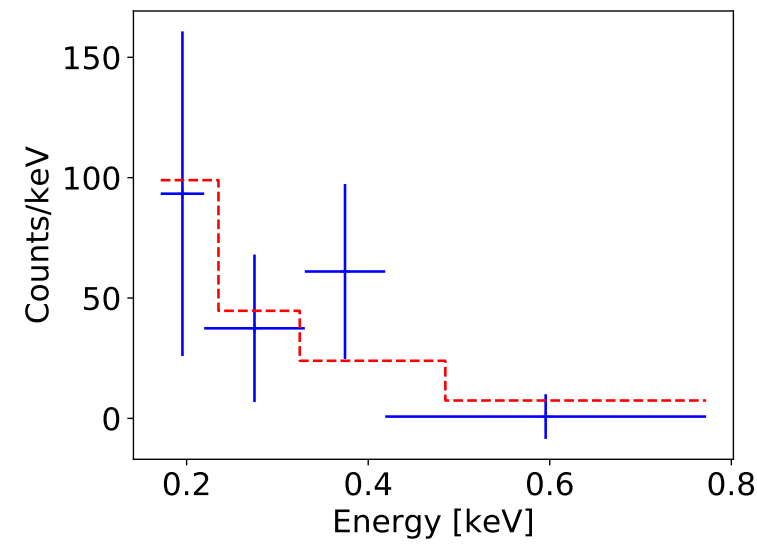

Fig. 4. XMM-Newton pn spectrum in 2006 with 10 counts per bin (solid blue) and best-fit model (red, dashed).

observation, for which no spectral information is available. By $\lambda_{s_{i}}(E M, T)$, we refer to the model source count rate as a function of emission measure and temperature, and $s_{i}$ and $b_{i}$ denote
Table 3. Counts detected in the $15^{\prime \prime}$ source region (SRC) and the $30-65^{\prime \prime}$ background annulus (BGA) in the individual EPIC instruments in the years 2000 and 2006.

\begin{tabular}{llrrr}
\hline \hline Instr. & Band & SRC & BGA & Net counts \\
\hline \multicolumn{5}{c}{ Year 2000} \\
MOS2 & soft & 3 & 18 & $1.75 \pm 1.76$ \\
MOS2 & hard & \multicolumn{4}{c}{8} & 18 & $6.75 \pm 2.84$ \\
& \multicolumn{4}{c}{ Year 2006} \\
MOS1 & soft & 8 & 52 & $4.45 \pm 2.87$ \\
MOS2 & soft & 13 & 42 & $10.03 \pm 3.63$ \\
pn & soft & 44 & 312 & $19.94 \pm 6.77$ \\
MOS1 & hard & 4 & 87 & $-1.94 \pm 2.10$ \\
MOS2 & hard & 3 & 93 & $-3.58 \pm 1.86$ \\
pn & hard & 16 & 183 & $1.89 \pm 4.13$ \\
\hline
\end{tabular}

the number of photons detected in the source and background region referred to by the observation and spectral band indicated by $i$. We used an inverse-rate prior on the background 


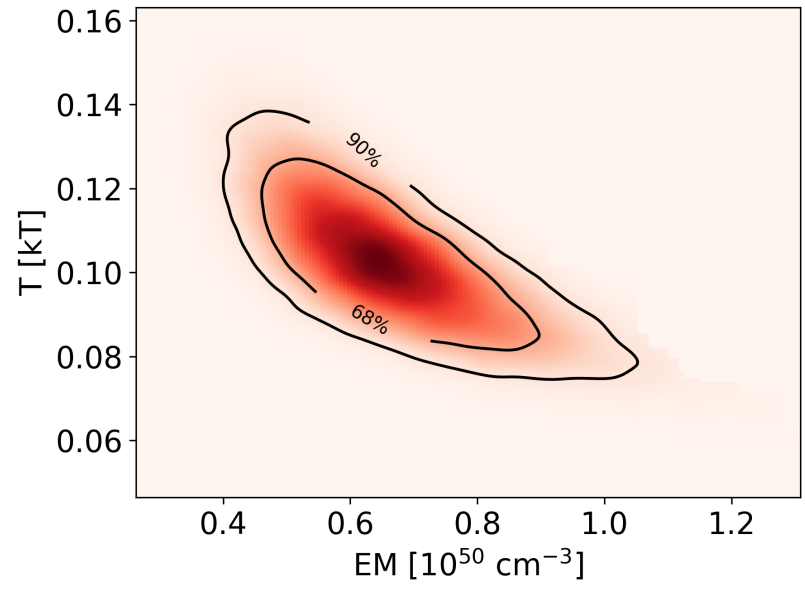

Fig. 5. Posterior probability distribution for emission measure and coronal temperature from combined analysis of all available observations of HD 209458

rate $\left(\lambda_{b}\right.$, Jaynes 1968). The factor $f_{a_{i}}$ is the area scaling between source and background and $I$ summarizes all information used in the posterior.

In Fig. 5 we show the posterior density along with the $68 \%$ and $90 \%$ highest probability density credibility regions. The marginal distributions yield a temperature estimate of $0.10(0.07,0.13) \mathrm{keV}\left(\right.$ or $\left.\log _{10}(T[\mathrm{~K}])=6.1(5.9,6.2)\right)$ and an emission measure of $0.7(0.4,0.9) \times 10^{50} \mathrm{~cm}^{-3}$, which corresponds to an X-ray luminosity of $8.4(5.8,11) \times 10^{26} \mathrm{erg} \mathrm{s}^{-1}$ in the $0.2-12 \AA$ band. In the $0.124-2.48 \mathrm{keV}$ band considered by Louden et al. (2017), we obtain an X-ray luminosity of $1.6(1.2,2) \times 10^{27} \mathrm{erg} \mathrm{s}^{-1}$ that is consistent with their results. In this band, the level of irradiation at the distance of the planet is $255(190,320) \mathrm{erg} \mathrm{cm}^{-2} \mathrm{~s}^{-1}$.

Neither the Chandra HRC-I nor XMM-Newton observations show significant short-term variability such as a flaring (see Sect. 2.1 and Fig. 6). The year 2000 XMM-Newton observation covers a planetary transit, which remains without noticeable effect owing to the low count rate. Nonetheless, the X-ray emission of HD 209458 may be variable on the timescales of years. In Table 4, we show the expected number of source counts along with the $90 \%$ credibility limits derived from the combined analysis of the observations; for convenience and reference, the estimated net counts are reproduced.

While we generally find good agreement, the year 2000 observation with the XMM-Newton MOS2 shows a conspicuous excess of hard-band photons; this excess is not seen during the year 2006 observation and is not accounted for by the model. This may, indeed, indicate a change in the coronal state of HD 209458 between the years 2000 and 2006. In the year 2000 observation, a hotter component may have been present similar to the maximum state of the Sun. However, we consider the supporting evidence too slim to draw a firm conclusion.

\section{Comparison to other stars}

According to Schmitt (1997), the minimum stellar X-ray surface flux of $F, G$, and $K$ type stars in the solar vicinity is $\approx 10^{4} \mathrm{erg} \mathrm{cm}^{-2} \mathrm{~s}^{-1}$. For HD 209458 this translates into a minimum X-ray luminosity of $8 \times 10^{26} \mathrm{erg} \mathrm{s}^{-1}$ in the ROSAT band, which is comparable to the value found in our analysis. Therefore, the activity level of HD 209458 is certainly at the lower end of the observed range. To check whether it may even be
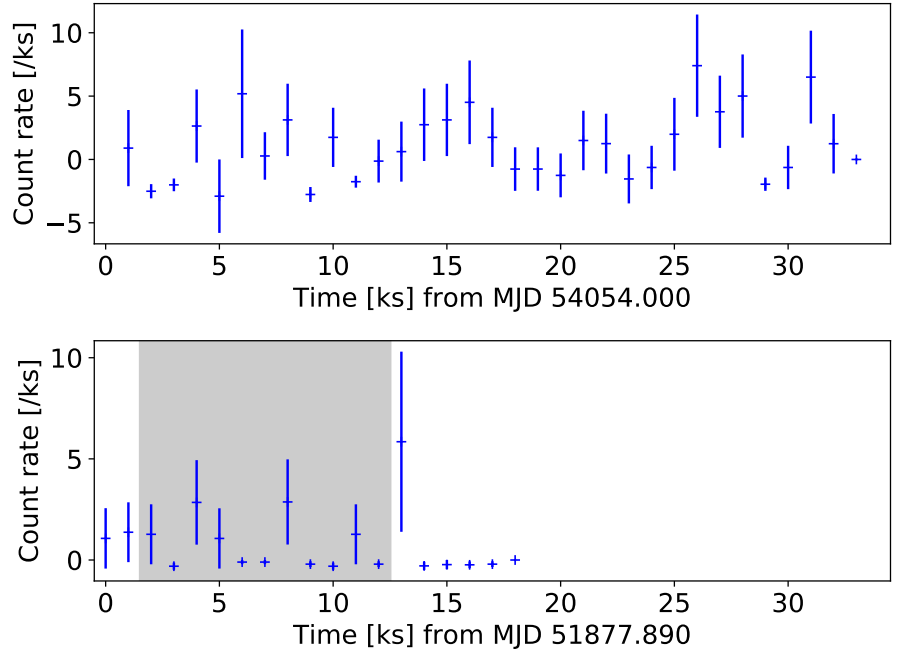

Fig. 6. Background-subtracted $0.15-1.5 \mathrm{keV} X M M$-Newton light curves with a binning of $1 \mathrm{ks}$. The top panel shows the $2006 \mathrm{pn}$ light curve and the bottom panel shows the 2000 MOS2 light curve. The shaded area in the bottom panel indicates the transit duration.

Table 4. Estimated net counts (replicated from Table 3) and expectation based on the combined modeling.

\begin{tabular}{|c|c|c|c|}
\hline Instr. & Band & Net counts & Model counts \\
\hline \multicolumn{4}{|c|}{ Year $2000($ XMM-Newton $)$} \\
\hline MOS2 & soft & $1.75 \pm 1.76$ & $1.17(0.8,1.6)$ \\
\hline MOS2 & hard & $6.75 \pm 2.84$ & $0.4(0.1,0.6)$ \\
\hline \multicolumn{4}{|c|}{ Year 2006 (XMM-Newton) } \\
\hline MOS1 & soft & $4.45 \pm 2.87$ & $3.2(2.1,4.3)$ \\
\hline MOS2 & soft & $10.03 \pm 3.63$ & $3.5(2.4,4.6)$ \\
\hline pn & soft & $19.94 \pm 6.77$ & $23.8(16.6,31)$ \\
\hline MOS1 & hard & $-1.94 \pm 2.10$ & $1(0.2,1.8)$ \\
\hline MOS2 & hard & $-3.58 \pm 1.86$ & $1(0.2,1.9)$ \\
\hline pn & hard & $1.89 \pm 4.13$ & $5.8(1.2,10.5)$ \\
\hline \multicolumn{4}{|c|}{ Year 2016 (Chandra) } \\
\hline HRC-I & - & $27.6 \pm 5.4$ & $29.4(20.8,37.7)$ \\
\hline
\end{tabular}

exceptional, for example, owing to its hot Jupiter, we now put the X-ray emission of HD 209458 in the context of other lowactivity stars.

\subsection{The $\alpha$ Centauri system}

The $\alpha$ Centauri system consists of a G2 V A component, a K1 V B component, and the red dwarf Proxima Centauri, orbiting the $\mathrm{AB}$ system in a wide orbit. With an age of $6.5 \pm 0.3 \mathrm{Gyr}$, the system is older than HD 209458 (Eggenberger et al. 2004). The $\mathrm{A}$ and $\mathrm{B}$ components have extensively been monitored using XMM-Newton (Robrade et al. 2012) and the Chandra HRC-I. A detailed analysis of the Chandra data was presented by Ayres (2014). Specifically, the problem of obtaining factors to convert HRC-I count rates into energy fluxes has been discussed by the author.

According to Ayres (2014), the B component shows an $8.1 \pm 0.2 \mathrm{yr}$ solar-like coronal activity cycle. The A component may have remained stuck in a Maunder-Minimum-like state until 2005, but appears to show an elevation in activity levels afterward, which may be indicative of a longer coronal cycle. 
In the low activity state of $\alpha$ Cen A, Ayres (2014) found that its corona is dominated by plasma at temperatures slightly above $1 \mathrm{MK}$, which is comparable to our result for HD 209458. The two coronae may therefore, be similar. Although the intrinsic energy resolution of the HRC-I is insufficient for a meaningful spectral analysis, some information on hardness is available. We tested whether the HRC-I spectrum of HD 209458 is consistent with that of the $\alpha$ Cen sources by comparing the photon energy distributions as given by the $\mathrm{PI}^{5}$ channel. To this end, we downloaded the 2005 HRC-I data of $\alpha$ Cen from the Chandra archive and extracted the photons corresponding to the $\alpha$ Cen A and $\mathrm{B}$ components; both are strong HRC-I sources so that background is negligible. In this period, the B component was observed close to its activity maximum, while the activity level of the A component was low. Using a Kolmogorov-Smirnov test, we compared the nominal photon energy distributions of the $\alpha$ Cen components with that of HD 209458. The resulting $p$-values are 0.77 for the A component and 0.07 for the B component. The test thus also provides no evidence against the hypothesis that $\alpha$ Cen $\mathrm{A}$ in its low-activity state observed in 2005 and HD 209458 show the same HRC-I spectrum.

Using the energy conversion factor provided by Ayres (2014, Eq. (2)), we estimated an X-ray luminosity of $(9 \pm 2) \times$ $10^{26} \mathrm{erg} \mathrm{s}^{-1}$ for HD 209458 in the $0.2-2 \mathrm{keV}$ band based on the HRC-I count rate alone. Again, this is entirely consistent with our previous analysis.

Compared to $\alpha$ Cen A in 2005, the X-ray luminosity of HD 209458 is about a factor of three higher. Both stars have similar radii of $1.224 R_{\odot}$ and $1.2 R_{\odot}$ and thus surface areas (Kervella et al. 2003; Boyajian et al. 2015), but $\alpha$ Cen A rotates with a period of $22.5 \pm 5.9$ d, i.e., more slowly than HD 209458 (Bazot et al. 2007). Although this is consistent with a lower activity level, both $\alpha$ Cen A and HD 209458 may of course show large, potentially cyclic variation in their coronal X-ray emission. While Chandra HRC-I is not well suited to resolve spectral variations in the coronal emission of HD 209458, changes in its luminosity could well be resolved by future observations.

\section{2. $51 \mathrm{Peg}$}

Similar to HD 209458, the G5V star 51 Peg also hosts a hot Jupiter (Mayor \& Queloz 1995). This star shows a sufficiently low activity level to qualify as a Maunder-Minimum candidate (Poppenhäger et al. 2009). Baliunas et al. (1995) present a Mount Wilson S-index time series of 51 Peg ranging from 1977 to 1991 , during which the star remained relatively stable with a mean S-index of 0.149 . Our more recent measurements with TIGRE yield a consistent S-index of $0.152 \pm 0.002$ (Mittag et al. 2016), providing no indication for a change in the activity state of 51 Peg.

Poppenhäger et al. (2009) studied the X-ray emission of 51 Peg and, among others, analyzed a Chandra HRC-I pointing. From their analysis, the authors concluded that the coronal temperature of $51 \mathrm{Peg}$ is $\lesssim 1 \mathrm{MK}$. Scaling the HRC-I count rate of 51 Peg reported by Poppenhäger et al. (2009) to the distance of HD 209458, we calculated a distance-scaled count rate of $0.42 \pm 0.1 \mathrm{ct} \mathrm{ks}^{-1}$. This implies that $51 \mathrm{Peg}$ is less luminous in X-rays than HD 209458 by about a factor of two, which is also consistent with the low coronal temperature derived by Poppenhäger et al. (2009).

\footnotetext{
Pulse Invariant.
}

\subsection{The Sun}

Because of the lack of appropriate instrumentation, continuous long-term studies of the broadband soft solar X-ray emission remain difficult. Peres et al. (2000) studied the Sun as an X-ray star, using data taken by the Yohkoh soft X-ray telescope (SXT), which remained, however, less sensitive at soft photon energies (Tsuneta et al. 1991), thereby increasing the uncertainty at the soft end of the spectrum.

Peres et al. (2000) simulated solar ROSAT spectra corresponding to the minimum and maximum of the solar cycle. During minimum, these authors found that the ROSAT spectrum can be fitted with a single $1 \mathrm{MK}$ component with an emission measure of $1.25 \times 10^{49} \mathrm{~cm}^{-3}$. In solar maximum, a hotter (2.6 MK) component appears and the cooler $1 \mathrm{MK}$ component increases in emission measure by about an order of magnitude. According to Peres et al. (2000), the solar X-ray luminosity in the ROSAT/PSPC band (here $0.1-3 \mathrm{keV}$ ) varies between about $0.23 \times 10^{27} \mathrm{erg} \mathrm{s}^{-1}$ and $4.4 \times 10^{27} \mathrm{erg} \mathrm{s}^{-1}$ between solar minimum and maximum.

These values are similar to those we found for HD 209458. According to our combined modeling of all available observations, the stellar corona is characterized by a temperature of around $1 \mathrm{MK}$ and an emission measure of (4-9) $\times 10^{49} \mathrm{~cm}^{-3}$. The coronal temperature of HD 209458 is thus comparable to that observed during solar minimum, but the emission measure corresponds to a value somewhere between solar minimum and maximum. Only a fraction of this can be attributed to the larger radius, leading to a $44 \%$ increase in the surface area compared to the Sun. During the year 2000 observation, HD 209458 may have shown a more active state that is perhaps similar to a solar maximum. However, the evidence here presented remains insufficient to draw such a conclusion.

\section{Conclusion}

We analyzed Chandra HRC-I and XMM-Newton data to determine the X-ray properties of HD 209458. Our HRC-I data unequivocally establish HD 209458 as an X-ray source. From the combined analysis of the available XMM-Newton and Chandra data, we determined a coronal temperature of about $1 \mathrm{MK}$ and and emission measure of $7(4-9) \times 10^{49} \mathrm{~cm}^{-3}$.

With respect to coronal temperature, HD 209458 appears to be comparable to the inactive Sun or $\alpha$ Cen A. We found that, in terms of HRC-I count rate, HD 209458 is more luminous than $\alpha$ Cen $\mathrm{A}$ in its low activity state. However, $\alpha$ Cen $\mathrm{A}$ and HD 209458 may of course both vary in their luminosity, possibly owing to cycles, which can only be studied with long-term monitoring programs. HD 209458 rotates faster than the Sun and $\alpha$ Cen A, which favors higher activity levels. This is also consistent with the distance-scaled HRC-I count rate of the MaunderMinimum candidate 51 Peg being lower than that of HD 209458. It appears, however, that HD 209458 is a faint X-ray emitter with respect to its rotation period of $14.4 \mathrm{~d}$. The relation between period and X-ray luminosity by Pizzolato et al. (2003), for instance, predicted an X-ray luminosity more than an order of magnitude higher than what we observed. Whether this bears some deeper meaning cannot be decided with the data at hand.

In short, HD 209458 is an inactive star in coronal X-rays that is comparable to the Sun itself. The coronal properties of HD 209458 derived in our analysis are consistent with those used by Louden et al. (2017) in their study and thus, demonstrate that the level of planetary atmospheric irradiation is sufficient to drive planetary evaporation at a rate of a few times $10^{10} \mathrm{~g} \mathrm{~s}^{-1}$. 
Acknowledgements. We thank the anonymous referee for a knowledgeable and helpful report. This work has made use of data from the European Space Agency (ESA) mission Gaia (http://www. cosmos. esa.int/gaia), processed by the Gaia Data Processing and Analysis Consortium (DPAC, http: //www . cosmos esa.int/web/gaia/dpac/consortium). Funding for the DPAC has been provided by national institutions, in particular the institutions participating in the Gaia Multilateral Agreement. S.C. and M.M. acknowledge support by UHH C.S. and M.S. acknowledge funding through DFG.

\section{References}

Arnaud, K. A. 1996, in Astronomical Data Analysis Software and Systems V, eds. G. H. Jacoby, \& J. Barnes, ASP Conf. Ser., 101, 17

Ayres, T. R. 2014, AJ, 147, 59

Baliunas, S. L., Donahue, R. A., Soon, W. H., et al. 1995, ApJ, 438, 269

Bazot, M., Bouchy, F., Kjeldsen, H., et al. 2007, A\&A, 470, 295

Bonfanti, A., Ortolani, S., \& Nascimbeni, V. 2016, A\&A, 585, A5

Boyajian, T., von Braun, K., Feiden, G. A., et al. 2015, MNRAS, 447, 846

Charbonneau, D., Brown, T. M., Latham, D. W., \& Mayor, M. 2000, ApJ, 529, L45

Daemgen, S., Hormuth, F., Brandner, W., et al. 2009, A\&A, 498, 567 del Burgo, C., \& Allende Prieto, C. 2016, MNRAS, 463, 1400

Eggenberger, P., Charbonnel, C., Talon, S., et al. 2004, A\&A, 417, 235

Foster, A. R., Ji, L., Smith, R. K., \& Brickhouse, N. S. 2012, ApJ, 756, 128

Gaia Collaboration (Brown, A. G. A., et al.) 2016a, A\&A, 595, A2

Gaia Collaboration (Prusti, T., et al.) 2016b, A\&A, 595, A1

Henry, G. W., Marcy, G. W., Butler, R. P., \& Vogt, S. S. 2000, ApJ, 529, L41

Isaacson, H., \& Fischer, D. 2010, ApJ, 725, 875

Jaynes, E. T. 1968, IEEE Transactions on Systems Science and Cybernetics, 4, 227

Kervella, P., Thévenin, F., Ségransan, D., et al. 2003, A\&A, 404, 1087

Knutson, H. A., Howard, A. W., \& Isaacson, H. 2010, ApJ, 720, 1569

Louden, T., Wheatley, P. J., \& Briggs, K. 2017, MNRAS, 464, 2396

Mamajek, E. E., \& Hillenbrand, L. A. 2008, ApJ, 687, 1264

Maxted, P. F. L., Serenelli, A. M., \& Southworth, J. 2015, A\&A, 577, A90
Mayor, M., \& Queloz, D. 1995, Nature, 378, 355

Mittag, M., Hempelmann, A., González-Pérez, J. N., Schmitt, J. H. M. M., \& Hall, J. C. 2011, in 16th Cambridge Workshop on Cool Stars, Stellar Systems, and the Sun, eds. C. Johns-Krull, M. K. Browning, \& A. A. West, ASP Conf. Ser., 448, 1187

Mittag, M., Schröder, K.-P., Hempelmann, A., González-Pérez, J. N., \& Schmitt, J. H. M. M. 2016, A\&A, 591, A89

Monet, D. G., Levine, S. E., Canzian, B., et al. 2003, AJ, 125, 984

Noyes, R. W., Hartmann, L. W., Baliunas, S. L., Duncan, D. K., \& Vaughan, A. H. 1984, ApJ, 279, 763

Peres, G., Orlando, S., Reale, F., Rosner, R., \& Hudson, H. 2000, ApJ, 528, 537

Pizzolato, N., Maggio, A., Micela, G., Sciortino, S., \& Ventura, P. 2003, A\&A, 397,147

Poppenhäger, K., Robrade, J., Schmitt, J. H. M. M., \& Hall, J. C. 2009, A\&A, 508, 1417

Raghavan, D., Henry, T. J., Mason, B. D., et al. 2006, ApJ, 646, 523

Robrade, J., Schmitt, J. H. M. M., \& Favata, F. 2012, A\&A, 543, A84

Rosen, S. R., Webb, N. A., Watson, M. G., et al. 2016, A\&A, 590, A1

Salz, M., Czesla, S., Schneider, P. C., \& Schmitt, J. H. M. M. 2016a, A\&A, 586, A75

Salz, M., Schneider, P. C., Czesla, S., \& Schmitt, J. H. M. M. 2016b, A\&A, 585, L2

Santos, N. C., Israelian, G., \& Mayor, M. 2004, A\&A, 415, 1153

Sanz-Forcada, J., Ribas, I., Micela, G., et al. 2010, A\&A, 511, L8

Sanz-Forcada, J., Micela, G., Ribas, I., et al. 2011, A\&A, 532, A6

Schmitt, J. H. M. M. 1997, A\&A, 318, 215

Schmitt, J. H. M. M., Schröder, K.-P., Rauw, G., et al. 2014, Astron. Nachr., 335, 787

Silva-Valio, A. 2008, ApJ, 683, L179

Tsuneta, S., Acton, L., Bruner, M., et al. 1991, Sol. Phys., 136, 37

Vidal-Madjar, A., Lecavelier des Etangs, A., Désert, J.-M., et al. 2003, Nature, 422, 143

Vidal-Madjar, A., Désert, J.-M., Lecavelier des Etangs, A., et al. 2004, ApJ, 604, L69

Winn, J. N., Noyes, R. W., Holman, M. J., et al. 2005, ApJ, 631, 1215

Wood, B. E., Redfield, S., Linsky, J. L., Müller, H.-R., \& Zank, G. P. 2005, ApJS, 159,118 